\title{
The Impact of a Panenterovirus Vp1 Assay on Our Perception of the Enterovirus Diversity Landscape of a Sample
}

\section{Abstract}

The number of independent emergence of recombinant circulating vaccine derived poliovirus serotype 2 (cVDPV2) lineages and the magnitude of the outbreak in Nigeria demonstrates the significance of enterovirus co-infection and its preponderance in the country. Despite this, besides polioviruses, little or no attention is given to enterovirus co-infections. More recently, a reversetranscriptase semi-nested polymerase chain reaction (RT-snPCR) assay for the recommended protocols for enterovirus surveillance. We previously showed that primers 292 and 222 (for which AN89 and AN88, respectively, are consensus degenerate hybrid oligonucleotide primers [CODEHOP] versions) mask the presence of enterovirus co-infections. We therefore ask whether the primers AN89 and AN88 used in this recently recommended RT-snPCR protocol, like primers 292 and 222, will also mask the presence of enterovirus co-infections. RNA was extracted from 30 archived samples (both clinical specimen and cell culture isolates) and the VP1 gene amplified using the WHO recommended RTsnPCR assay and modifications that included the primers 187, 188 and 189 for which primer 292 (and consequently AN89) is a consensus. Amplicons were sequenced and isolates identified. Our results showed that primers AN89 and AN88 also mask enterovirus co-infection and inclusion of the primers 187, 188 and 189 allowed the resolution of such mixed isolates. Consequently, expanding the recommended RT-snPCR protocol to include primers 187, 188 and 189 will enable us better detect enterovirus co-infection.

Keywords: Enterovirus species resolution; Nix protocol; Nigeria; Acute flaccid paralysis; Diversity landscape direct detection of enteroviruses from clinical specimen was added to the WHO

\begin{tabular}{|c|}
\hline Research Article \\
\hline Volume 4 Issue 3 - 2016 \\
\hline Temitope Oluwasegun Cephas Faleye ${ }^{1,2}$, \\
\hline Moses Olubusuyi Adewumi ${ }^{1}$, Simbiat Adeola \\
\hline Kareem $^{3}$, Yetunde Olubunmi Adesuyan ${ }^{3}$, \\
\hline Fadekemi Ayodele Fapohunda ${ }^{3}$, Samson \\
\hline Tunde Fasanya3, Temitope Jimeto ${ }^{3}$, Osaze \\
\hline Emmanuel Lawrence ${ }^{3}$, Abolaji Abiodun \\
\hline Obembe $^{3}$ and Johnson Adekunle Adeniji ${ }^{1,4 *}$ \\
\hline 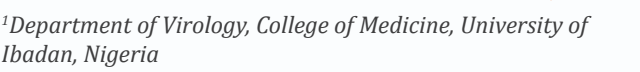 \\
\hline $\begin{array}{l}\text { 2Department of Microbiology, Faculty of Science, Ekiti State } \\
\text { University, Nigeria }\end{array}$ \\
\hline $\begin{array}{l}{ }^{3} \text { Department of Science Laboratory Technology, Faculty of } \\
\text { Science, Ekiti State University, Nigeria }\end{array}$ \\
\hline${ }^{4}$ WHO National Polio Laboratory, University of Ibadan, Nigeria \\
\hline \multirow{3}{*}{$\begin{array}{l}\text { *Corresponding author: Johnson Adekunle ADENIJI, WHO } \\
\text { National Polio Laboratory, Department of Virology, College } \\
\text { of Medicine, University of Ibadan, Ibadan, Oyo State, Nigeria, } \\
\text { Tel: +234802 } 300 \text { 9059; Email: adek1808@yahoo.com }\end{array}$} \\
\hline \\
\hline \\
\hline $\begin{array}{l}\text { Received: November 29, } 2016 \text { | Published: December 29, } \\
2016\end{array}$ \\
\hline
\end{tabular}

Abbreviations: cVDPV2: Circulating Vaccine Derived Poliovirus Serotype 2; RT-snPCR: Reverse-Transcriptase Semi-Nested Polymerase Chain Reaction; UTRs: Untranslated Regions; ORF: Open Reading Frame; CODEHOP: Consensus Degenerate Hybrid Oligonucleotide Primers; E7: Echovirus 7

\section{Introduction}

Enteroviruses are members of the genus Enterovirus in the family Picornaviridae, order Picornavirales. Within this genus are 12 species, four (Enterovirus species A - Enterovirus species D [EV-A to EV-D]) of which used to be called 'human enteroviruses'. Within these four (EV-A to EV-D) species are over 100 serotypes, three (3) of which are polioviruses (type members of the genus and specifically belonging to enterovirus species C) (www. picornaviridae.com). It has been estimated that in the United States alone, about $10-15$ million enterovirus infections occur annually [1]. However, less than $10 \%$ of these infections manifest with clinical presentations [2]. Clinically manifest enterovirus infections range from very mild ones like common cold [3], to serious ones like intrauterine transmission with fatal outcome [4] and paralysis or myelitis [3].

The enterovirus virion is naked and has a diameter of 28-30nM.
The genome is a single-stranded, positive-sense, $\sim 7.5 \mathrm{~kb}$ RNA molecule. It has one open reading frame (ORF) that is flanked on both sides (5' and 3') by untranslated regions (UTRs) and a poly-A tail at the $3^{\prime}$ end. The ORF is translated into a polyprotein that is auto-catalytically cleaved into three (P1, P2 and P3) polyproteins. The P1 polyprotein is further cleaved into four (VP1-VP4) proteins that form the capsid (structural proteins). The P2 and P3 polyproteins are also further cleaved into 2A- 2C, and 3A-3D respectively. These seven proteins $(2 \mathrm{~A}-2 \mathrm{C}$ and $3 \mathrm{~A}-3 \mathrm{D})$ are the non-structural proteins and are crucial in enterovirus replication.

The enterovirus genome has been described as 'a mosaic' [5] because of the frequency of recombination and the observation that a single genome could undergo multiple recombination events. Recombination is one of the mechanisms used by enteroviruses for evolution and often result in the emergence of new clades with variant phenotype(s), sometimes including increased transmissibility and pathogenicity [5]. Enterovirus coinfection is however, a prerequisite for recombination between different enterovirus genomes.

Between 2005 and 2011, Nigeria experienced the biggest outbreak of circulating vaccine derived poliovirus (cVDPV) ever 
recorded [6]. cVDPVs are vaccine-derived poliovirus strains that unlike the non-pathogenic vaccine strains have regained their pathogenicity and have increased transmissibility [7]. During the Nigeria cVDPV outbreak, period there were 403 confirmed cases which were resolved into 23 independent emergence. Only 7 of the cVDPVs isolated during this were not recombinants. All the others were recombinants with structural region of OPV origin and non-structural region of nonpolio enterovirus species $\mathrm{C}$ origin. The magnitude of the recombinant circulating vaccine derived poliovirus serotype 2 (cVDPV2) outbreaks in Nigeria [6] and the number of independent emergence of recombinant cVDPV2 lineages demonstrates the significance of enterovirus coinfection, and its preponderance in Nigeria. Despite this, besides the resolution of poliovirus serotypes present in an isolate [8], little or no attention is given to nonpolio enteroviruses present in poliovirus isolates and more specifically, nonpoliio enterovirus co-infections.

Recently, the WHO recommended direct detection and identification of enterovirus from clinical specimen [9] as an addition to the previously recommended cell culture based strategy for enterovirus surveillance $[10,11]$. To accomplish this, the Nix et al. [12] RT-snPCR protocol (subsequently referred to in this manuscript as the Nix protocol) was adopted. Since its first description in 2006 [12], several independent groups [13-15] have used the Nix protocol and confirmed its sensitivity.

Besides the four primers (AN32-AN35) used for cDNA synthesis, the Nix protocol [12] uses four other primers for the RT-snPCR assay. For the first round PCR assay, primers 224 and 222 are used. For the second round PCR assay, primers AN89 and AN88 are used. Primers AN88 and AN89 are consensus degenerate hybrid oligonucleotide primers (CODEHOP) versions of primers 222 and 292, respectively [12]. Primer 292, however, was first described in 2003 [16] as a consensus of primers 187, 188 and 189.

We recently showed that, in cases where there is evidence that a sample contains more than one enterovirus species, VP1 amplification and sequencing using the primers 292 and 222 would sometimes amplify only one of the serotypes present [17]. In this study, we ask whether the primers AN89 and AN88 used in the Nix protocol have similar shortcoming. We further investigated whether the inclusion of the composite primers (187, 188 and 189), for which primer 292 (and consequently AN89) are consensus, would help in the resolution of such mixed isolates along species lines. Our results show that primers AN89 and AN88, like primers 292 and 222, tend to show the presence of one enterovirus even in cases where members of more than one species are present in the sample. Furthermore, the results of this study show that the inclusion of the primers 187, 188 and 189 is useful in the resolution of mixed isolates.

\section{Methods}

\section{Samples}

Considering the preponderance of enterovirus co-infection in Nigeria, manifest in the emergence of several recombinant cVDPV2 lineages [6], thirty (30) samples were selected and analyzed using the study algorithm schematically represented in Figure 1. Ten (10) samples each were randomly selected from three different category of samples (Table 1 ).

\section{Sample set 1}

Sample sets 1 consists of ten (10) (numbers 1-10; Table 1) suspensions of faecal material collected from apparently healthy school aged children. These samples were collected in 2014 as part of a study previously conducted by our group [15]. The 10 samples in set 1 had been previously positive for enteroviruses (using the Nix protocol) and the identity/ serotype of the enterovirus in each sample, determined (Table 1).

\section{Sample set 2}

The second set of ten (10) samples (numbers 11-20; Table 1) are also faecal suspensions made from faeces of apparently healthy school aged children. These samples were collected in 2014 as part of a previous study [17] and were negative for enteroviruses when previously screened. The only exception was sample 17 (Table 1) which was previously positive for an enterovirus screen but the sequence data could not be used to identify the resident enterovirus due to the presence of multiple peaks in the electropherogram.

\section{Sample set 3}

The third set of ten (10) samples (numbers 21-30; Table 1) consist of cell culture isolates recovered between 2010 and 2015 from acute flaccid paralysis (AFP) cases and an environmental sample (Table 1). Except for samples 28 and 30 which had been previously identified using a species $\mathrm{C}$ specific assay (under development in our laboratory) to contain poliovirus serotype 1 and Coxsackievirus A13 respectively, no attempt has been previously made to identify the isolate resident in these samples.

\section{RNA extraction and cDNA synthesis}

RNA extraction and cDNA synthesis were done using Jena Bioscience RNA extraction kit and Script cDNA synthesis kit (Jena Bioscience, Jena, Germany) respectively. These were done according to the manufacturer's instructions. However, during cDNA synthesis, instead of random hexamers, primers AN32, AN33, AN34 and AN35 [12] were used.

\section{Enterovirus VP1 gene Semi-nested PCR assay}

Primers were made in $100 \mu \mathrm{M}$ concentrations and PCR was done in $30 \mu \mathrm{L}$ reactions with the first round PCR containing $0.3 \mu \mathrm{L}$ each of primers 224 and 222 [12], $6 \mu \mathrm{L}$ of Red load Taq, $10 \mu \mathrm{L}$ of cDNA and $13.4 \mu \mathrm{L}$ of RNase free water. Thermal cycling was done using a Veriti thermal cycler (Applied Biosystems, California, USA) at $94^{\circ} \mathrm{C}$ for 3 minutes followed by 45 cycles of $94^{\circ} \mathrm{C}$ for 30 seconds, $42^{\circ} \mathrm{C}$ for 30 seconds and $60^{\circ} \mathrm{C}$ for 60 seconds with ramp of $40 \%$ from $42^{\circ} \mathrm{C}$ to $60^{\circ} \mathrm{C}$. This was then followed by $72^{\circ} \mathrm{C}$ for 7 minutes and held at $4^{\circ} \mathrm{C}$ till terminated.

Four different second round PCR assays were carried out using the first round PCR product as template, with similar thermal cycling conditions except for the extension time that was reduced to 30 seconds. Primer AN88 [12] was used as the reverse primer in all the four second round PCR screens. However, the forward primers were, AN89, 189, 187 and 188 for the panenterovirus (PE), species A (EV-A), B (EV-B), and C and D (EV-C \& D) screens respectively [9]. Subsequently, PCR products were resolved on $2 \%$ agarose gel stained with ethidium bromide, and viewed using a UV transilluminator. 


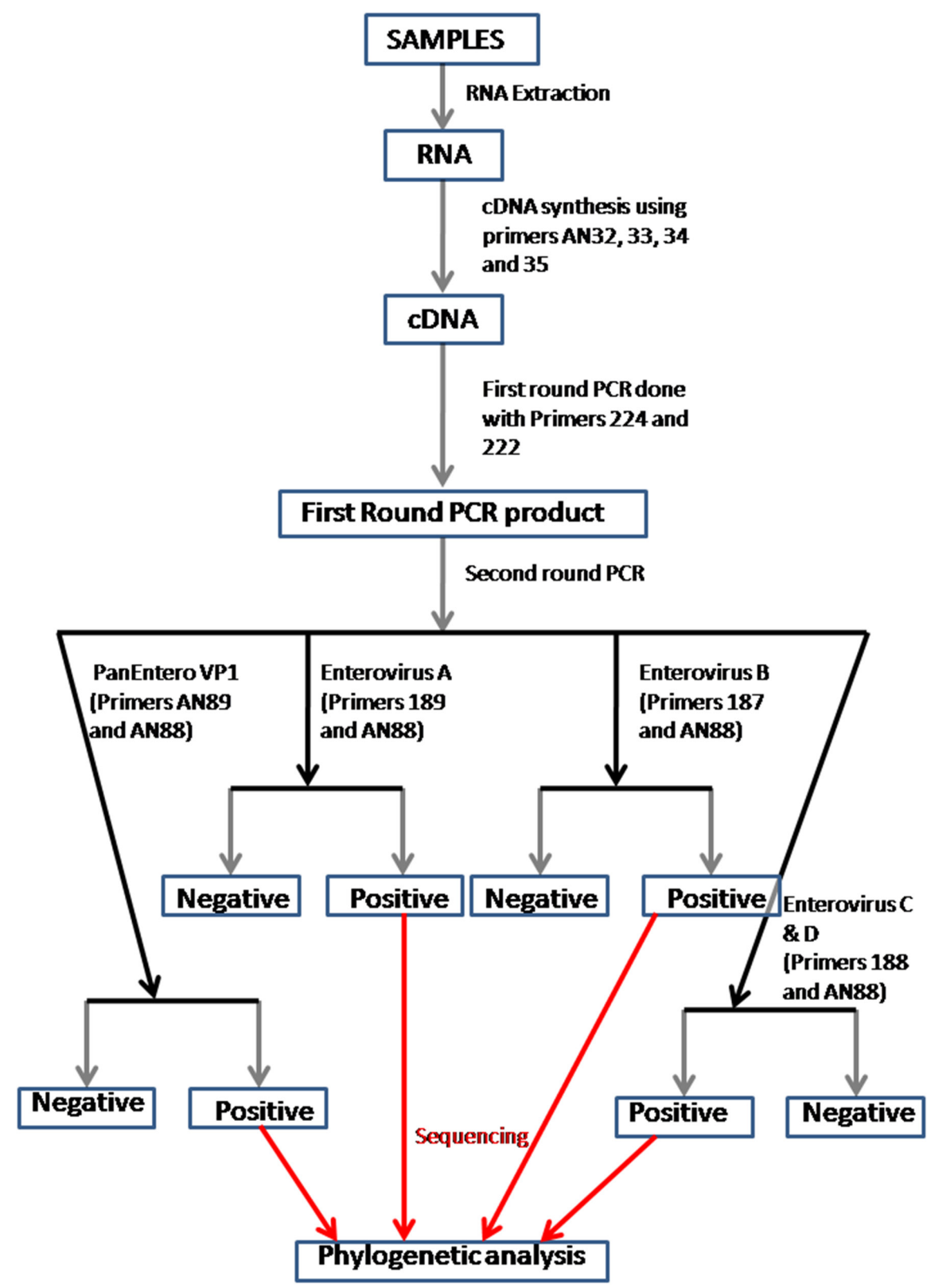

Figure 1: Schematic representation of the algorithm followed in this study. 
Table 1: Enterovirus Identification using the Pan Ent protocol and the species specific assays (modifications).

\begin{tabular}{|c|c|c|c|c|c|c|c|c|c|}
\hline \multirow{2}{*}{\multicolumn{6}{|c|}{ Sample Information }} & \multicolumn{4}{|c|}{ Results } \\
\hline & & & & & & \multirow{2}{*}{$\begin{array}{c}\text { PanEnt } \\
\text { Repeat Pan } \\
\text { ENT } \\
\text { VP1 }\end{array}$} & \multicolumn{3}{|c|}{ Modifications } \\
\hline $\mathbf{S} / \mathbf{N}$ & Sample Type & $\begin{array}{c}\text { Year of } \\
\text { Collection }\end{array}$ & $\begin{array}{c}\text { Enterovirus } \\
\text { Previously } \\
\text { Detected }\end{array}$ & $\begin{array}{l}\text { Identity } \\
\text { by VP1 }\end{array}$ & $\begin{array}{c}\text { ENT } \\
\text { Species }\end{array}$ & & EV-A & EV-B & EV-C \& D \\
\hline 1 & Faecal Suspension & 2014 & Yes & CVA1 & $\mathrm{C}$ & CVA1 & CVA1 & - & CVA1 \\
\hline 2 & Faecal Suspension & 2014 & Yes & CVA8 & $\mathrm{A}$ & CVA8 & CVA8 & - & CVA8 \\
\hline 3 & Faecal Suspension & 2014 & Yes & EVB80 & $\mathrm{B}$ & EVB80 & - & - & \\
\hline 4 & Faecal Suspension & 2014 & Yes & CVA20 & $\mathrm{C}$ & CVA20 & CVA20 & - & CVA20 \\
\hline 5 & Faecal Suspension & 2014 & Yes & EVA71 & $\mathrm{A}$ & EV71 & EV71 & - & EV71 \\
\hline 6 & Faecal Suspension & 2014 & Yes & EVA71 & A & EV71 & EV71 & - & Unexp \\
\hline 7 & Faecal Suspension & 2014 & Yes & CVA4 & A & CVA4 & CVA4 & EVB88 & Unexp \\
\hline 8 & Faecal Suspension & 2014 & Yes & CVA3 & A & - & - & - & - \\
\hline 9 & Faecal Suspension & 2014 & Yes & E32 & $\mathrm{B}$ & E32 & - & E32 & - \\
\hline 10 & Faecal Suspension & 2014 & Yes & EVC99 & $\mathrm{C}$ & - & - & - & - \\
\hline 11 & Faecal Suspension & 2014 & No & NA & - & - & - & - & - \\
\hline 12 & Faecal Suspension & 2014 & No & NA & - & - & - & - & - \\
\hline 13 & Faecal Suspension & 2014 & No & NA & - & - & - & - & - \\
\hline 14 & Faecal Suspension & 2014 & No & NA & - & - & - & - & - \\
\hline 15 & Faecal Suspension & 2014 & No & NA & - & - & - & - & - \\
\hline 16 & Faecal Suspension & 2014 & No & NA & - & - & - & - & - \\
\hline 17 & Faecal Suspension & 2014 & Yes & Unexp & - & $\mathrm{E} 2$ & - & E2 & - \\
\hline 18 & Faecal Suspension & 2014 & No & NA & - & - & - & - & - \\
\hline 19 & Faecal Suspension & 2014 & No & NA & - & Unexp & - & - & - \\
\hline 20 & Faecal Suspension & 2014 & No & NA & - & Phage & Unexp & - & Unexp \\
\hline 21 & Isolate (AFP-RD) & 2010 & No & UNT & - & E14 & - & E14 & - \\
\hline 22 & Isolate (AFP-RD) & 2010 & No & UNT & - & E11 & - & E11 & - \\
\hline 23 & Isolate (AFP-RD) & 2010 & No & UNT & - & - & - & - & - \\
\hline 24 & Isolate (AFP-RD) & 2012 & No & UNT & - & - & - & - & - \\
\hline 25 & Isolate (AFP-RD) & 2012 & No & UNT & - & E7 & - & E7 & - \\
\hline 26 & Isolate (AFP-RD) & 2012 & No & UNT & - & E3 & - & E3 & - \\
\hline 27 & Isolate (AFP-RD) & 2013 & No & UNT & - & E7 & E7 & E7 & Unexp \\
\hline 28 & Isolate (AFP-RD) & 2013 & Yes & PV1 & $\mathrm{C}$ & - & - & E6 & - \\
\hline 29 & Isolate (ES-RD) & 2015 & No & UNT & - & CVB5 & - & CVB5 & - \\
\hline 30 & Isolate (ES-MCF 7) & 2012 & Yes & CVA13 & $\mathrm{C}$ & - & CVA13 & - & - \\
\hline
\end{tabular}

AFP: Acute Flaccid Paralysis; UNT: Untyped; RD: Rhabdomyosarcoma Cell line; Unexp: Unexploitable; ES: Environmental Surveillance; EV: Enterovirus MCF 7: MCF 7 Cell Line; E: Echovirus; NA: Not Applicable; CV: Coxsackievirus; PV: Poliovirus 


\section{Nucleotide sequencing}

All amplicons were shipped to Macrogen Inc, Seoul, South Korea, for purification and sequencing. The primers used for the second round PCR were also used for sequencing. The species and serotype of the sequenced isolates were first identified using the enterovirus genotyping tool [18].

\section{Nucleotide sequence accession numbers}

All the isolates whose sequences are being determined in this study for the first time have been deposited in GenBank under the accession numbers KT972735 - KT972743.

\section{Results}

\section{Enterovirus VP1 gene Semi-nested PCR assay}

In this study, the three (3) different sample sets were analysed using the same first round but four (4) different second round PCR assays (Figures 1). For sample set 1, all the samples except samples 8 and 10 had the expected band size and were consequently considered positive for the PE-PCR screen. As regards the EV-A PCR screen, all the samples except samples 3, 8, 9 and 10 were positive. For the EV-B PCR screen, only samples 7 and 9 were positive while samples 1, 2, 4, 5, 6 and 7 were positive for the EV-C\&D-PCR screen.

For sample set 2, all the samples except samples 17, 19 and 20 were negative for the PE-PCR screen. For the EV-A PCR screen all the samples except samples 20 were negative. For the EV-B PCR screen, only sample 17 was positive and only sample 20 was positive for the EV-C\&D PCR screen.

For sample set 3, all the samples except samples 23, 24, 28 and 30 were positive for the PE-PCR screen. For the EV-A PCR screen all the samples were negative except for samples 27 and 30. For the EV-B PCR screen, the result was the same as that of the PEPCR screen and only sample 27 was positive for the EV-C\&D PCR screen.

\section{Enterovirus identification}

As regards sample set 1 , the PE-PCR isolates from samples 1 , $2,3,4,5,6,7$ and 9 were identified as CVA1, CVA8, EVB80, CVA20, EV71, EV71, CVA4 and E32 respectively. The EVA-PCR isolates from samples 1, 2, 4, 5, 6 and 7 were identified as CVA1, CVA8, CVA20, EV71, EV71 and CVA4 respectively. The EV-A PCR isolates from sample 8 could not be identified because the electropherogram had multiple peaks and was consequently unexploitable. The EV-B PCR isolates from samples 7 and 9 were identified as EVB88 and E32 respectively. The EV-C\&D PCR isolates from samples 1, 2, 4, 5, were CVA1, CVA8, CVA20 and EV71 respectively. The EVC\&D PCR isolates from samples 6 and 7 could not be identified because their electropherograms had multiple peaks and were consequently unexploitable (Table 1 ).

As regards sample set 2, the PE-PCR isolate from sample 17 was identified as E2. That from sample 19 could not be identified because the electropherogram had multiple peaks and was consequently unexploitable. While, the PE-PCR isolate from sample 20 was not similar to any enterovirus in the EGT database.
A BLAST search however revealed it to be most similar to an enterobacteriophage baseplate gene. The EV-A PCR and EV-C\&D PCR isolates from sample 20 were both unexploitable due to presence of multiple peaks in their electropherograms. However, the single EV-B PCR isolate (sample 17) was identified as E2 (Table 1).

With respect to sample set 3 , the PE-PCR isolates from samples 21, 22, 25, 26, 27 and 29 were identified as E14, E11, E7, E3, E7 and CVB5 respectively. The EV-A PCR isolates from samples 27 and 30 were identified as E7 and CVA13 respectively. The EV-B PCR isolates from samples 21, 22, 25, 26, 27, 28 and 29 were identified as E14, E11, E7, E3, E7, E6 and CVB5 respectively. The single EV-C\&D PCR isolate from sample 27 was unexploitable due to presence of multiple peaks in its electropherogram.

\section{The PE-PCR versus the Modifications}

The PE-PCR assay and the modifications gave about the same result in all but four (4) of the samples analyzed (Table 1). In sample 3, the PE-PCR assay showed the presence of EVB80 while the modifications could not detect the presence of any enterovirus. In sample 7, the PE-PCR assay showed the presence of CVA4 while the modifications showed the presence of both CVA4 and EVB88. In samples 28 and 30, the PE-PCR assay could not detect the presence of any enterovirus while the modifications showed the presence of E6 and CVA13, respectively.

\section{Discussion}

In this study the PE-PCR assay was able to detect the presence of an enterovirus serotype (EVB80) that even the modifications could not detect. Furthermore, the PE-PCR assay detected almost all the sequences independently amplified by the modifications (EV-A, EV-B and EV-C\& D PCR assays) (Table 1). The results of this study therefore showed that the PE-PCR assay [12] as recently recommended by the WHO [9] for direct detection and identification of enteroviruses from clinical specimen, is indeed robust and sensitive. However, there were few exceptions (Table 1). The PE-PCR assay failed to detect the presence of PV1 \& E6 in sample 28, and CVA13 in sample 29. These serotypes where however, independently detected by an EV-C screen (being developed in our laboratory, unpublished) the EV-B and EV-A screens, respectively. Considering that the same first round product was used as template for the three (PE, EV-A and EV-B) second round assays concerned, it appears that this false negative result from the PE-PCR assay is an indication that AN89 and AN88 primers need to be further improved to increase their sensitivity.

Despite the shortcomings highlighted above, being a consensus of primers 187, 188 and 189, primer AN89 has acquired the sensitivity to function in the capacity of the three. As regards enterovirus detection and identification, this is a significant upgrade as it reduces the number of assays to be run and consequently saves time and resources, while still providing the information necessary for both molecular epidemiology and diagnosis. However, the downside of being a consensus primer, for AN89 (and 292 by extension), is loss of the resolving power of 187, 188 and 189, especially in cases of co-infection. For example, primers AN89 and AN88 identified CVA4 in sample 7 (Table 1). The presence of CVA4 in sample 7 was confirmed by primers 189 
and AN88 (Table 1). However, at the same time, primers 187 and AN88 showed that sample 7 also contained EVB88 (Table 1).

It is pertinent to note that this is the first time EVB88 is being detected in Nigeria and had we relied on primers AN89 and AN88 alone, we would have missed the presence of EVB88 in this sample. Therefore, considering the preponderance of enterovirus co-infections, it might be necessary to re-think and balance our desire for a panenterovirus detection assay with our ability to define the enterovirus serotype diversity of any sample of interest.

The results of this study show that though the primers 189 and 188 (recommended for EV-A and EV-C \& D respectively) are suggested to be different in their preference for different enterovirus species [9], in our laboratory, they appear to have the same preferences (Table 1). So far, in our laboratory, both primers amplify both EV-A and EV-C. In fact, it appears as though primer 189 amplifies everything primer 188 amplifies and it does it better (Table 1). Furthermore, a case was also found in which primer 189 amplified an EB (echovirus 7; sample 27 (Table 1)). It is therefore important to note that as valuable as these primers are for resolution of enterovirus mixtures, it should not be assumed that they always amplify along the suggested [9] species lines. Consequently, the results of these assays, without sequencing, should not be used in isolation to determine the species to which an isolate belongs.

In this study, we were able to identify isolates that were recovered in cell culture as way back as 2010 and had been kept at $-20^{\circ} \mathrm{C}$ since isolation (Table 1). Ordinarily, such isolates, in our setting, are usually unrecoverable in cell culture due to incessant power failure, the consequent repeated freeze-thaw cycles and the resultant loss in virion infectivity. However, our ability to still identify these isolates using the Nix protocol and its modifications, as described in this study, shows that enterovirologists in resource limited settings might be able to analyse archived isolates in their facilities and generate valuable sequence data that will help us better understand the global dynamics of enteroviruses.

Contrary to our observation with isolates first recovered in cell culture, the situation was different with faecal suspensions stored at $-20^{\circ} \mathrm{C}$ for about six (6) months (Table 1 ). We were only able to recover enteroviruses from $80 \%(8 / 10)$ of faecal suspensions that were previously confirmed to contain such. This suggest that, given the difficulty associated with maintaining a stable freezing temperature in our environment, the titre of virus in such samples had dropped below the detection limit of the assays within six month. The fact that we could now type sample 17, which in our previous analysis (six months before [15]) was unexploitable due to the presence of multiple-peaks in its electropherogram, might confirm the drop in titre explanation. This therefore suggest that in resource limited settings, and especially, places where power supply is epileptic, it might be better to first subject samples to cell culture and store recovered isolates.

Though the strategy suggested above might lengthen the storage time of enterovirus isolates recovered and our ability to revisit such isolates for re-analysis, prior subjection to cell culture might result in cell line bias and consequently, influence our perception of the enterovirus diversity landscape of the sample as previously shown [17-22]. Subsequent to the completion of this study, we used the same algorithm described in this study to directly screen faecal suspensions from AFP cases and their corresponding isolates on RD cell line. We found that the enterovirus isolate recovered on RD cell line does not fully describe the enterovirus serotype diversity landscape of the same faecal suspension. For example, in one instance, the RD isolate was echovirus 7 (E7) while species specific screens of the original faecal suspension showed that the sample also contained E14 and EVC99 (unpublished data).

In another instance, the PE-PCR assay showed the RD isolate was EVB75 while EVB77 was detected in the original faecal suspension (unpublished data). Thus, cell culture bias alongside the inability (or otherwise) to detect and resolve enterovirus coinfections as described in this study, show the impact of detection algorithm on our perception of the enterovirus diversity landscape of any specimen. It is therefore important that detection algorithms are developed that take into account the biases and limitations of the different components of the algorithm. Such algorithms will enable us get a much more balanced picture of the enterovirus diversity landscape of any specimen and might be our first steps in resolving the conundrums of enteroviruses and their association with an array of disease conditions.

\section{Acknowledgements}

The authors would like to thank the members of staff of the WHO National Polio Laboratory in Ibadan, Nigeria, for providing us with most of the isolates in sample set 3 .

\section{Ethical Approval}

The samples analyzed in this study were collected as part of previous independent studies but were anonymized before use in this study. Therefore, this article does not contain any studies with human participants performed by any of the authors.

\section{Author Contributions}

1) Study Design (FTOC, AMO, AJA)

2) Acquisition of reagents, laboratory and data analysis (All Authors)

3) Wrote the first draft of the Manuscript (FTOC)

4) Revised the Manuscript (All Authors)

5) Read and Approved the Final Draft (All Authors)

\section{References}

1. Strikas RA, Anderson LJ, Parker RA (1986) Temporal and geographic patterns of isolates of nonpolio enteroviruses in the United States, 1970-1983. J Infect Dis 153(2): 346-351.

2. Nathanson N, Kew OM (2010) From Emergence to Eradication: The Epidemiology of Poliomyelitis Deconstructed. Am J Epidemiol 172(11): 1213-1229.

3. Tapparel C, Siegrist F, Petty TJ, Kaiser L (2013) Picornavirus and enterovirus diversity with associated human disease. Infect Genet Evol 12(3): 505-521.

4. Tassin M, Martinovic J, Mirand A, Peigue-Lafeuille H, Picone O, et al. (2014) A case of congenital Echovirus 11 infection acquired early in pregnancy. J Clin Virol 59(1): 71-73. 
5. Combelas N, Holmblat B, Joffret ML, Colbère-Garapin F, Delpeyroux F (2011) Recombination between Poliovirus and Coxsackie A Viruses of Species C: A Model of Viral Genetic Plasticity and Emergence. Viruses 3(8): 1460-1484.

6. Burns CC, Shaw J, Jorba J, Bukbuk D, Adu F, et al. (2013) Multiple Independent Emergences of Type 2 Vaccine-Derived Polioviruses during a Large Outbreak in Northern Nigeria. J Virol 87(9): $4907-$ 4922.

7. Burns CC, Diop OM, Sutter RW, Kew OM (2014) Vaccine-Derived Polioviruses. The Journal of Infectious Diseases 210(S1): S283-S293.

8. Kilpatrick DR, Yang CF, Ching K, Vincent A, Iber J, et al. (2009) Rapid Group-, Serotype-, and Vaccine Strain-Specific Identification of Poliovirus Isolates by Real-Time Reverse Transcription-PCR Using Degenerate Primers and Probes Containing Deoxyinosine Residues. J Clin Microbiol 47(6): 1939-1941.

9. World Health Organisation (2015) Enterovirus surveillance guidelines: Guidelines for enterovirus surveillance in support of the Polio Eradication Initiative, Geneva, Switzerland.

10. World Health Organisation (2003) Guidelines for environmental surveillance of poliovirus circulation. Geneva, Switzerland.

11. World Health Organisation (2004) Polio laboratory Manual. ( $4^{\text {th }}$ edn), Geneva, Switzerland.

12. Nix WA, Oberste MS, Pallansch MA (2006) Sensitive, Seminested PCR Amplification of VP1 Sequences for Direct Identification of All Enterovirus Serotypes from Original Clinical Specimens. J Clin Microbiol 44(8): 2698-2704.

13. Rahimi P, Tabatabaie H, Gouya MM, Mahmudi M, Musavi T, et al. (2009) Direct identification of non-polio enteroviruses in residual paralysis cases by analysis of VP1 sequences. J Clin Virol 45(2): 139-141.

14. Sadeuh-Mba SA, Bessaud M, Joffret ML, Endegue Zanga MC, Balanant J, et al. (2014) Characterization of Enteroviruses from non-human primates in cameroon revealed virus types widespread in humans along with candidate new types and species. PLoS Negl Trop Dis 8(7): e3052.
15. Faleye T0, Adewumi MO, Coker BA, Nudamajo FY, Adeniji JA (2016) Direct Detection and Identification of Enteroviruses from Faeces of Healthy Nigerian Children Using a Cell-Culture Independent RTSeminested PCR Assay. Adv Virol 2016: 1412838.

16. Oberste MS, Nix WA, Maher K, Pallansch MA (2003) Improved molecular identification of enteroviruses by RT-PCR and amplicon sequencing. J Clin Virol 26(3): 375-377.

17. Faleye TO, Adeniji JA (2015) Enterovirus species B bias of RD cell line and its influence on enterovirus diversity landscape. Food Environ Virol 7(4): 390-402.

18. Kroneman A, Vennema H, Deforche $K$, v d Avoort H, Peñaranda S, et al. (2011) An automated genotyping tool for enteroviruses and noroviruses. J Clin Virol 51(2): 121-125.

19. Sadeuh-Mba SA, Bessaud M, Massenet D, Joffret ML, Endegue MC, et al. (2013) High frequency and diversity of species $C$ enteroviruses in Cameroon and neighbouring countries. J Clin Microbiol 51(3): 759770.

20. Adeniji JA, Faleye TO (2014a) Isolation and identification of enteroviruses from sewage and sewage contaminated water in Lagos, Nigeria. Food Environ Virol 6(2): 75-86.

21. Adeniji JA, Faleye TO (2014b) Impact of Cell Lines Included in Enterovirus Isolation Protocol on Perception of Nonpolio Enterovirus Species C Diversity. J Virol Methods 207: 238-247.

22. Adeniji JA, Faleye TO (2015) Enterovirus C strains circulating in Nigeria and their contribution to the emergence of recombinant circulating vaccine-derived polioviruses. Arch Virol 160(3): 675-683. 